\title{
Philosophiques
}

\section{Réalisme, esprit réaliste, antiréalisme}

\section{Mathieu Marion}

Volume 45, numéro 1, printemps 2018

URI : https://id.erudit.org/iderudit/1048626ar

DOI : https://doi.org/10.7202/1048626ar

Aller au sommaire du numéro

Éditeur(s)

Société de philosophie du Québec

ISSN

0316-2923 (imprimé)

1492-1391 (numérique)

Découvrir la revue

Citer ce document

Marion, M. (2018). Réalisme, esprit réaliste, antiréalisme. Philosophiques, 45(1), 261-270. https://doi.org/10.7202/1048626ar d'utilisation que vous pouvez consulter en ligne.

https://apropos.erudit.org/fr/usagers/politique-dutilisation/ 


\title{
Réalisme, esprit réaliste, antiréalisme
}

\author{
MATHIEU MARION \\ Département de philosophie \\ Université du Québec à Montréal
}

We are swimming in the deep waters of metaphysics.

How can we attain the shore?

Michael Dummett

Dans L'adresse du réel ${ }^{1}$, Jocelyn Benoist poursuit sa réflexion sur la phénoménologie, amorcée dans Les limites de l'intentionnalité et continuée entre autres dans Éléments de philosophie réaliste et Concepts ${ }^{2}$, réflexion dont un des thèmes centraux est le "problème du réalisme". On pourrait même parler de l'élaboration d'une "phénoménologie réaliste ", en se gardant bien de comprendre cette expression dans le sens où l'entendait, par exemple, Roman Ingarden ${ }^{3}$, et ce pour des raisons que j'aimerais tirer au clair dans ce bref commentaire.

Cette tâche est quelque peu compliquée par le fait que Jocelyn Benoist développe ses idées en critiquant du même souffle des conceptions rivales; je pense entre autres à sa critique de John McDowell dans Concepts ${ }^{4}$ ou encore, ici même, à sa critique du "nouveau réalisme" promu par Markus Anders et Maurizio Ferraris ${ }^{5}$. Mon intention n'est cependant pas de juger la pertinence de ses critiques. Benoist jongle dans les premières pages de L'adresse $d u$ réel avec des thèmes chers à ces derniers, tels que celui d'un "retour de la métaphysique » ou encore le grand récit d'une "philosophie européenne" renaissant à la faveur de la disparition des philosophies "analytique " et "continentale ", qui laisserait littéralement entrevoir une "clôture de ce qui aura été la parenthèse kantienne dans l'histoire de la philosophie ${ }^{6} »$. Ce récit impliquerait donc la disparition de la philosophie "analytique» - du moins en tant que Kampfbegriff dans le contexte français -, mais cela n'empêche nullement

1. J. Benoist, L'adresse du réel, Paris, Vrin, 2017.

2. J. Benoist, Les limites de l'intentionnalité, Paris, Vrin, 2005, Éléments de philosophie réaliste, Paris, Vrin, 20I I et Concepts. Introduction à l'analyse, Paris, Éditions du Cerf, 20 I I.

3. Voir, par exemple, les textes traduits dans R. Ingarden, La controverse idéalismeréalisme, Paris, Vrin, $200 \mathrm{I}$.

4. Voir sur ce point R. Moati, «Jocelyn Benoist: Concepts », disponible à l'adresse:

http://www.actu-philosophia.com/Jocelyn-Benoist-Concepts-258.

5. M. Anders (hrg.), Der Neue Realismus, Berlin, Suhrkamp, 20I4; et M. Ferraris, Manifesto del nuovo realismo, Rome, Laterza, $20 \mathrm{I} 2$.

6. J. Benoist, L'adresse du réel, op. cit., p. I3 et I6. 
Jocelyn Benoist de faire un usage essentiel de travaux de philosophes réputés "analytiques". En grossissant le trait, on pourrait justement décrire son projet comme une tentative de repenser la phénoménologie en accord avec le «contextualisme» de Charles Travis en philosophie du langage ${ }^{7}$. Ce qui passe à la trappe, pour ainsi dire, dans cette présumée "clôture de la parenthèse kantienne", ce serait plutôt les approches rivales, que sont les sémantiques représentationnelles ou cognitives, tandis que "l'antiréalisme » basé sur la sémantique des preuves est évacué comme n'étant qu'une mauvaise solution de rechange à ces dernières ${ }^{8}$. Selon moi, c'est aller trop vite, et c'est sur ce terrain que je voudrais engager la discussion. Mon intention n'est pas de défendre les sémantiques représentationnelles ou cognitives, mais de montrer que la position que développe Benoist dans son livre est plus proche de l'antiréalisme qu'il ne veut bien l'admettre?.

Selon la définition courante en philosophie analytique, être « réaliste» à propos d'un domaine quelconque ou, dans une version plus «linguistique ", une classe de phrases comme, par exemple, celles portant sur le passé ou sur ce que Benoist appelle au chapitre V les «impossibilia», revient à soutenir conjointement deux thèses, soit: a) qu'il existe des faits ou entités spécifiques à ce domaine, et b) que leur existence soit objective et indépendante de notre esprit. Benoist souscrit pleinement à ces deux thèses. Ces deux passages suffisent à démontrer le soutien de Benoist à la thèse (a), si par «transcendance» on reconnaît l'existence de faits ou entités spécifiques:

Or avec la dimension du référent, c'est un autre sens du réalisme qui se fait jour, fondé sur la reconnaissance d'une transcendance par rapport au sens, auquel seul le sens donne accès. Être réaliste, c'est précisément faire droit à une telle transcendance ${ }^{10}$.

[...] être réaliste en un certain sens plein du terme, qui réclame plus que la simple validité épistémique, c'est exiger qu'en toute forme de connaissance une transcendance véritable soit rencontrée ${ }^{11}$.

7. Peut-être faudrait-il parler de «l'occasionnalisme» de Travis, puisque son concept clé est celui de la «sensibilité à l'occasion» (occasion-sensitivity), et qu'il rejette explicitement ce qu'on appelle le "contextualisme». Voir, par exemple, C. Travis, Occasion Sensitivity. Selected Essays, Oxford, Oxford University Press, 2008, p. I4. Pourtant, Travis se prête au jeu dans «Insensitive Semantics» (Mind \& Language, vol. 2 I, 2006, p. 39), en se déclarant représentant d'un «contextualisme radical ", ce qui porte à confusion.

8. J. Benoist, L'adresse du réel, op. cit., p. 35-36.

9. Par "antiréalisme ", je réfère à un type de position mis de l'avant pour la première fois par Michael Dummett, tout en reconnaissant que certains aspects de sa propre position sont aujourd'hui considérés désuets, entre autres en ce qui concerne le rôle central joué par la loi du tiers exclu. On parle justement d'antiréalisme «dummettien» pour distinguer ses positions d'autres formes d'antiréalisme.

10. Ibid., p. 34 .

11. Ibid., p. 36. 
Par ailleurs, Benoist reprend à son compte dans ce même passage le concept «d'objectivité» mis de l'avant par Frege, avec son exemple bien connu de la superficie de la mer du Nord, au $\mathbb{2} 6$ de Fondements de l'arithmétique $^{12}$; il soutient donc aussi la thèse (b).

Pourtant, Benoist qualifie la tradition de la sémantique référentielle, appelée ici "période sémantique ", d'antiréaliste:

$[\mathrm{L}]$ a philosophie analytique, quand elle atteint son âge classique $[\ldots]$ a connu une phase très fortement anti-réaliste. Cet anti-réalisme est caractéristique de sa période sémantique, où, même si elle prétendait être réaliste, elle mettait essentiellement le monde à distance, comme ce à quoi nous pourrions accéder qu'en y référant. Comme s'il y avait toujours la distance d'un acte et/ou d'un dispositif de référence entre le monde et nous ${ }^{13}$.

Cette caractérisation de la sémantique référentielle me semble fautive, la référence n'impliquant dans une telle sémantique aucune "distance", «acte» ou «dispositif» entre le monde et le langage (et non le «nous»). Je ne veux cependant pas débattre de cette question, mais simplement noter l'usage particulier de l'opposition entre « réalisme» et «antiréalisme », car la sémantique référentielle est justement tenue, comme Benoist le note luimême, pour "réaliste", car elle est agencée avec les thèses (a)-(b) ci-dessus. Au chapitre V, Benoist qualifie de façon tout aussi surprenante "d'antiréaliste » un type de position sur les impossibilia apparenté à celle de Meinong:

Un élément déterminant dans la fixation du sens de ce qu'on appelle «réalisme» est le sort fait à la notion d'«impossibilité». En la matière, l'antiréalisme a choisi. Il est, depuis toujours, tenant de la maximalisation du possible, auquel il voudrait ne pas voir de limites ${ }^{14}$.

Ainsi, l'anti-réaliste aime-t-il à se payer d'impossible. Rien ne lui est, a priori, impossible ${ }^{15}$.

Benoist ne qualifie pas explicitement Meinong d'antiréaliste, mais l'exemple qu'il choisit pour sa discussion des impossibilia, l'objet «carré rond ", est révélateur. Bien sûr, il sait très bien que Meinong est tenu pour «le plus réaliste des philosophes ${ }^{16}$ ", mais pour lui ce "réalisme» est "dans une certaine mesure dévoyé» :

Le réalisme ne peut consister à tenir que des choses qui n'existent pas existent, même en un sens dilué ou déplacé de l' «existence ${ }^{17}$ ».

12. G. Frege, Les fondements de l'arithmétique, Paris, Éditions du Seuil, I969, p. I $52-$ I 55 .

13. J. Benoist, L'adresse du réel, op. cit., p. 60.

14. Ibid., p. I69.

15. Ibid., p. I70.

16. Ibid., p. 66.

17. Ibid., p. 66-67. 
Cette dernière phrase montre bien jusqu'à quel point Benoist n'entend pas par «réalisme» ce qu'on entend à partir des thèses (a)-(b). D'où proviendrait cet usage particulier? Il semble que soit en cause ici la fidélité à une «attitude » ou un «esprit réaliste », au sens où Cora Diamond a introduit cet usage en philosophie ${ }^{18}$. Cet usage est repris explicitement par Sandra Laugier, pour qui l'expression "être réaliste", doit être comprise comme dans l'injonction "Soyez réaliste! ${ }^{19}$, à l'opposé d'une attitude qu'on pourrait qualifier "d'idéaliste» comme celle, par exemple, d'un politicien qui ne se soucierait guère, aux yeux de ses adversaires, des conséquences de la dette.

L'opposition est voulue ici entre un usage plus proprement philosophique et l'usage "ordinaire» réputé non philosophique. En philosophie, le terme "réalisme» est diversement défini en termes de débats contre une variété de positions adverses, comme le "nominalisme », «l'idéalisme », etc., et c'est l'opposition entre le "réalisme» au sens des thèses (a)-(b) ci-dessus et «l'antiréalisme» qui est ici remise en cause. En français comme en anglais, le terme "réalisme» recouvre plusieurs sens, dont le plus ancien est justement philosophique, trouvant sa source dans les querelles du nominalisme au Moyen-Âge ${ }^{20}$. Selon un autre usage, des plus répandus, "réalisme" désigne en art et en littérature l'attachement à reproduire la réalité sans fard ou «sans idéal ${ }^{21}$. Cette définition n'est pertinente pour nous que sous un seul aspect par ailleurs fort intéressant, soit l'idée de «l'attention aux détails » ou aux "particularités». En simplifiant grandement, on pourrait décrire le projet de Jocelyn Benoist comme une tentative d'ajuster la phénoménologie au "contextualisme» de Charles Travis ${ }^{22}$, et on peut justement concevoir l'attention aux contextes ou occasions d'usage sous un mode apparenté à ce qu'on pourrait décrire comme «l'attention aux détails» en peinture. On pourrait donc condamner les adversaires de ce «contextualisme» pour la pauvreté de leur description du langage, donc leur manque de «réalisme».

18. Diamond introduisit la notion «d'esprit réaliste» dans «Le réalisme et l'esprit réaliste ", réimprimé dans C. Diamond, L'esprit réaliste: Wittgenstein et la philosophie de l'esprit, Paris, P.U.F., 2004, p. 55-IO2; voir tout particulièrement p. 56-59.

19. S. Laugier, Du réel à l'ordinaire. Quelle philosophie du langage aujourd'hui ?, Paris, Vrin, I999, p. I4. Benoist reprend explicitement ce sens. Voir J. Benoist, Éléments de philosophie réaliste, op. cit., p. I I.

20. Pour la langue anglaise, voir l'entrée "Realism» de Raymond Williams dans Keywords. A Vocabulary of Culture and Society, nouvelle édition, Oxford, Oxford University Press, 2015, p. 198-202.

21. Cet usage est aussi discuté dans C. Diamond, L'esprit réaliste: Wittgenstein et la philosophie de l'esprit, op. cit., p. 57.

22. Peut-être faudrait-il parler de «l'occasionnalisme» de Travis, puisque son concept clé est celui de la "sensibilité à l'occasion» (occasion-sensitivity), et qu'il rejette explicitement ce qu'on appelle le "contextualisme». Voir, par exemple, C. Travis, Occasion Sensitivity. Selected Essays, Oxford, Oxford University Press, 2008, p. I4. Pourtant, Travis se prête au jeu dans «Insensitive Semantics» (Mind \& Language, vol. 2 I, 2006, p. 39), ce qui porte à confusion. 
Je ne voudrais pas ouvrir un débat sur ce point, mais simplement noter qu'il va tout à fait de soi qu'une philosophie du langage ou une théorie linguistique, qui ne pourrait rendre compte que d'une toute petite partie de la variété des usages du langage manquerait de "réalisme» et serait tout simplement sans intérêt. Or la question n'est jamais tout à fait là, car tous veulent être "réalistes", et les tenants des sémantiques référentialistes et cognitives ont d'autres explications des supposés contre-exemples (y compris ceux qu'on décrit parfois comme des "Travis cases»), et le débat reste tout à fait ouvert entre Travis et ses adversaires ${ }^{23}$, ceux-ci cherchant plutôt à expliquer (à tort ou à raison) les autres usages comme étant fondés sur l'usage référentiel du langage ou, à tout le moins comme pour Grice, comme ne remettant pas en cause la centralité de ce dernier ${ }^{24}$. En d'autres termes, bien qu'on puisse y souscrire, l'idée de «l'esprit réaliste " n'a pas à mon avis — ça se discute - le tranchant requis pour véritablement jouer par ellemême un rôle critique.

Un contraste avec ce qui est «imaginaire» ou seulement de l'ordre de «l'apparence» est lui aussi impliqué dans l'usage «ordinaire». En anglais, le contraste entre "réel» et «imaginaire» est présent dès les débuts à la fois chez Shakespeare et Hobbes ${ }^{25}$. Et lorsque Cora Diamond invoque un unique passage de Wittgenstein à l'appui de sa thèse que ce dernier aurait incarné l'esprit réaliste ${ }^{26}$, elle ne semble pas s'apercevoir que celui-ci implique justement ce contraste ${ }^{27}$. Tout au plus pourrions-nous voir derrière cet usage une

23. Pour ne donner que deux exemples, voir H. Cappelen \& E. Lepore, Insentitive Semantics. A Defense of Semantic Minimalism and Speech Act Pluralism, Oxford, Blackwell, 2005, et F. Recanati, Truth-Conditional Pragmatics, Oxford, Clarendon Press, 2010.

24. Grice est explicite là-dessus en plusieurs endroits, par exemple dans H. P. Grice, «Reply to Richards », dans R. Grandy \& R. Warner (dir.), Philosophical Grounds of Rationality Oxford, Clarendon Press, I986, p. 59 ou Studies in the Way of Words, Cambridge MA, Harvard University Press, I989, p. 4.

25. Chez Shakespeare: "Is't real that I see" (All's Well That Ends Well, V, iii); chez Hobbes: "not Imaginary, but Reall" (New Leviathan, III, xxxiv). Ces exemples sont tirés de R. Williams, Keywords, op. cit., p. I98. L'image du philosophe assis dans son fauteuil (armchair philosophy) est aujourd'hui condamnée de toutes parts. J. L. Austin la condamnait déjà, par exemple, dans la discussion générale à Royaumont (La philosophie analytique, Paris, Éditions de Minuit, I962, p. 335). Encore faut-il considérer que l'attention à la variété des usages fasse de soi-même autre chose qu'un philosophe assis dans son fauteuil d'un type particulier. Selon moi, ce contraste indique qu'on devrait plutôt éviter d'opposer les concepts déployés par les armchair philosophers et leur usage dans le langage "ordinaire", en présupposant que ce dernier soit le fin mot, comme si la frontière entre les deux n'était pas à la fois mobile et poreuse.

26. C. Diamond, L'esprit réaliste: Wittgenstein et la philosophie de l'esprit, op. cit., p. 58 .

27. Diamond cherche à élucider le sens de la phrase de Wittgenstein «Pas l'empirisme, et pourtant le réalisme en philosophie », mais ne s'appuie dans ce texte (voir note précédente) que sur un seul autre passage des Bemerkungen über die Grundlagen der Mathematik (Francfort, Suhrkamp, I984, III, $\mathbb{S} 76$ ), où Wittgenstein utilise les contrastes entre «réaliste » (realis- 
injonction à ne pas se préoccuper de ce qui importe, et non de chimères. C'est là, il me semble, le sens mis de l'avant par Diamond et Laugier, selon lequel être «réaliste» veut plutôt dire quelque chose «tenir compte de la réalité » ou de «comment sont les choses», pour parler comme Benoist ${ }^{28}$. Or, outre l'injonction, qui ressemblerait de loin à une nouvelle version de l'argument par le «common sense» - par exemple, que le «bon sens» dicterait que les impossibilia n'existent pas -, il est difficile de voir dans l'adoption d'un «esprit réaliste» la prémisse d'un argument pour la supériorité d'une parmi de nombreuses approches rivales, prétendant toutes expliquer les mêmes faits.

S'il entend bien par «réalisme» l'idée de tenir compte de "comment sont les choses ", Benoist évite cet écueil, en définissant plutôt le terme à partir d'Aristote. Il aime citer ce passage de la Métaphysique, qu'il commente en disant qu'Aristote y énonce "l'idée fondamentale d'un véritable réalisme ${ }^{29} »$ :

En effet, tu n'es pas blanc parce que nous pensons vraiment que tu es blanc, mais, nous qui affirmons cela, nous disons vrai parce que tu es blanc ${ }^{30}$.

Pour Benoist cette remarque met de l'avant l'indépendance de la réalité, en accord avec (b), et sa priorité par rapport à la vérité et notre connaissance. La vérité est donc "fondée sur la réalité", elle exprime "comment sont les choses". Bien qu'on parle d'un usage philosophique, on retrouve donc ici le sens soi-disant «ordinaire» de «réalisme»:

Dans ces termes nous tenons l'idée fondamentale d'un véritable réalisme (à opposer à un réalisme prétendu) : à savoir celle d'un enracinement ontologique de la vérité. Ce qui est vrai ou non dépend essentiellement de comment sont les choses - nommons cela: réalité. À défaut de cette exigence, il n’y a pas de réalisme ${ }^{31}$.

Le passage par «l'ordinaire» apparaît donc relever de l'artifice, en ce sens qu'il ne permet pas de se placer dans un nouvel angle d'attaque, différent de celui d'Aristote, pour tenter de résoudre ou dissoudre des problèmes philosophiques. En effet, ou bien on s'en tient à la définition courante en termes de

tische) et «tangible» (greifbar) d'un côté, et, de l'autre, «obscurantisme» (Obskurantismus) et «brumeux» (Schattenhaft).

28. J. Benoist, L'adresse du réel, p. 6r. Étymologiquement, le mot «réalisme» vient du latin «realis", dérivé lui-même de "res", qui a pris dans le contexte légal la signification de «chose", l'anglais ayant conservé cette origine dans l'expression "real estate».

29. Ibid., p. 6I.

30. Met. $\Theta$, Iо, I05 Ib, 6-9 (traduction de M.-P. Duminil \& A. Jaulin). Passage cité (dans une autre traduction) par Benoist dans L'adresse du réel, p. 36 et 6I. Cette idée est aussi présente dans la célèbre définition de la vérité au livre $\Gamma$, que, curieusement, Benoist ne cite pas: «En effet, dire que l'être n'est pas ou que le non-être est, c'est faux; dire que l'être est et que le non-être n'est pas, c'est vrai» (Met. $\Gamma$, 7, IOI I b25).

31. J. Benoist, L'adresse du réel, p. 6I. 
(a)-(b), ou bien on adopte cette nouvelle définition, mais alors on se retrouve à soutenir une position de type "antiréaliste». Le cas des impossibilia est ici exemplaire, puisque la position défendue par Benoist au chapitre $\mathrm{V}$ revient justement à rejeter (a) pour ces supposées entités. Appeler sa position «réaliste » et qualifier celle de Meinong "d'antiréaliste» en vertu de cette nouvelle définition ne fait selon moi que masquer l'aspect "antiréaliste» de ses positions. Il eût été plus approprié de qualifier Meinong de "non réaliste», afin d'éviter l'ambiguité dans l'usage du mot " antiréalisme ${ }^{32}$.

S'il salue le «retour de la métaphysique», Benoist reste critique du «nouveau réalisme», qu'il juge lié à un «besoin exacerbé de métaphysique ${ }^{33}$ » et à un « libéralisme ontologique extrême et pour ainsi dire hors contrôle $e^{34}$ ». Sa réaction en est donc une de prudence, qui n'est justement pas sans rappeler celle de l'antiréaliste. En effet, Dummett distinguait l'idée d'une thèse antiréaliste sur la signification d'une portée générale (qu'on lui attribue généralement) de son programme de recherche, ce dernier consistant à évaluer le réalisme (défini en termes de (a)-(b)) au cas par cas, d'une classe de phrases à l'autre ${ }^{35}$. Ce qui implique que nous pourrions très bien adopter une forme de "réalisme " pour telle ou telle classe de phrases, comme celles portant sur le passé, tout en étant «antiréaliste» à propos des impossibilia. Benoist ne semble pas dire autre chose:

Sans doute faut-il plutôt considérer que chaque forme discursive ouvre son propre registre de transcendance, et que la question du réalisme mathématique, par exemple - et encore faudrait-il distinguer entre les différentes parties de la mathématique - ne se pose pas exactement dans les mêmes termes que celle du réalisme physique - et encore là, quelle physique? — ni que celui du sens commun ${ }^{36}$.

Le « retour de la métaphysique » s'oppose par ailleurs à mon avis bien plus à la philosophie du langage de Davidson, qui pensait qu'une analyse réduisant la signification à une théorie tarskienne de la vérité aurait aussi l'avantage d'évacuer la métaphysique, alors que Dummett, loin de prétendre les dissoudre, proposait d'utiliser plutôt la théorie de la signification comme simple tremplin pour les aborder ${ }^{37}$. En effet, Davidson pensait que l'écriture

32. Cette ambiguïté est présente, par exemple, dans les occurrences aux pages 36 et I 69 .

33. J. Benoist, L'adresse du réel, p. 65.

34. Ibid., p. 70.

35. M. A. E. Dummett, The Seas of Language, p. 462-465.

36. J. Benoist, L'adresse du réel, p. 36.

37. Voir, par exemple, l'introduction à The Logical Basis of Metaphysics (Oxford, Clarendon Press, I99I, p. I-I9) intitulée «Metaphysical Disputes about Realism ». Dummett reste certes un "philosophe du langage» en ceci qu'il ne croit pas que nous puissions aborder les problèmes métaphysiques directement, sans détour par l'analyse du langage. Cela ressort par exemple dans sa critique du "nominalisme", dont "l'hostilité » envers les objets abstraits menant à la tentative d'expurger du langage toute référence à ceux-ci procède de la mauvaise manière, en tranchant le problème métaphysique d'abord, par ce qui ne pourrait être que 
de la Convention T de Tarski, "La neige est blanche" est vrai si et seulement si la neige est blanche", permettrait de faire l'économie de la notion de «correspondance» et donc de la métaphysique (des "faits", "états de choses ", etc.) qu'elle présuppose, comme par exemple avec une formulation telle que: "La neige est blanche" est vrai si et seulement si il y a correspondance avec le fait (ou l'état de choses) la neige est blanche». Dans une entrevue en 2003, Davidson mettait les choses au clair en des termes non équivoques:

De nombreux philosophes qui se disent eux-mêmes réalistes insistent sur une forme de vérité-correspondance [une théorie qui dit en gros qu'un énoncé est rendu vrai par un fait dans le monde], mais puisque je pense que cela n'a aucun sens, en ce sens je ne suis pas réaliste ${ }^{38}$.

Dummett écrivait pour sa part ceci dès I959, dans "Truth »:

[N]ous avons aujourd'hui abandonné la théorie de la vérité-correspondance. [...] Mais cette théorie exprime néanmoins une caractéristique importante du concept de vérité qui n'est pas exprimée par la loi <Il est vrai que $P$ si et seulement si $P>$, et que nous avons jusqu'ici laissée de côté, à savoir qu'un énoncé est vrai seulement s'il y a quelque chose dans le monde en vertu de quoi il est vrai. Bien que nous n'acceptions plus la théorie de la vérité-correspondance, nous restons, an fond, des réalistes ${ }^{39}$.

À la fois Davidson et Dummett utilisent ici le mot «réaliste» dans un sens équivalent à celui de Benoist. En fait, Davidson confirme son point de vue sur la "période sémantique ", cité ci-dessus. Mais il ne fait pas de doute que Dummett est ici bien plus proche de lui. Encore une fois, décrire la position de Davidson comme étant «non réaliste» aurait évité toute confusion inutile.

L'idée qu'il doit y avoir quelque chose dans le monde qui rend nos énoncés vrais est même connue aujourd'hui sous le nom de "principe $\mathrm{C}$ ", que lui donna plus tard Dummett ${ }^{40}$. Dans le cas des énoncés mathématiques, les intuitionnistes que ce sont les preuves et non des entités "platoniciennes »

\footnotetext{
«superstition» (M. A. E. Dummett, Truth and Other Enigmas, Londres, Duckworth, I978, p. xlii.) Ayant toujours eu une attitude respectueuse envers la métaphysique, Dummett pensait simplement que l'étude du langage nous permettrait éventuellement d'en solutionner les problèmes de manière satisfaisante, et non les «dissoudre " ou les «éliminer ", comme chez Wittgenstein, Carnap et bien d'autres. Il peut y avoir quelque chose d'étrange à dire que nous en sommes seulement à débattre de la façon dont nous devrions nous y prendre pour solutionner les problèmes de la métaphysique, mais ce n'est pas la même chose que de dire que ce sont de faux problèmes appelés à disparaître.
}

38. Entrevue disponible à l'adresse: [http://www.philosophersmag.com/interviews/27donald-davidson-interviewed].

39. M. A. E. Dummett, Philosophie de la logique, op. cit., p. 62.

40. M. A. E. Dummett, The Seas of Language, op. cit., p. 52. 
— autrement dit ici les preuves font office de «truth-makers» ${ }^{41}$. On ne peut pas reprocher à Benoist, qui ne fait qu'allusion à ce débat ${ }^{42}$, de ne pas parler de ce dont il ne parle pas, mais il qualifie en passant le choix entre ces deux options de "forme de chantage ${ }^{43}$ ", tandis que son insistance sur la thèse (b) et son refus d'un concept de vérité "épistémique» vont directement à l'encontre du point de vue intuitionniste. Lorsque Benoist écrit:

La vérité vaut indépendamment de la capacité de certains agents cognitifs de l'appréhender. Il s'agit là d'un point de grammaire: d'élucidation de notre usage du mot "vrai»; et non d'une quelconque thèse métaphysique quant à l'existence «transcendante» d'entités que nous appellerions des "vérités ${ }^{44}$ ».

On voudrait lui répondre que c'est aussi une remarque de "grammaire " (philosophique) et "d'élucidation », que de reconnaître cette particularité des énoncés mathématiques, plutôt que d'insister - en contradiction avec le passage cité plus haut à ce sujet — sur un traitement uniforme.

Dernier point, Benoist oppose à une lecture «chosiste» de la distinction de Frege entre sens et référence, qui débouche sur un "platonisme ", une lecture «normativiste» pour laquelle le sens n'est qu'une prise sur les choses, qui permet "contextuellement de les qualifier comme étant comme ceci ou comme cela ${ }^{45}$ ». Selon moi, cette lecture s'accommode très bien dans le domaine des mathématiques d'une lecture du «sens» comme «algorithme » et de la "référence» comme la «valeur», que le calcul nous permet d'obtenir ${ }^{46}$. Après tout, pour savoir que "I $27 \times 229=29083$ ", il faut effectuer un calcul. Se contenter de dire que les deux membres de l'équation référent à un même objet, c'est rater l'essentiel.

Mon intention n'est bien sûr pas de rallier Benoist à l'antiréalisme, ni non plus le critiquer pour ne pas l'avoir fait, mais simplement de faire voir qu'à bien y penser l'antiréalisme n'est pas une fausse solution de remplacement sans intérêt à ce qu'il appelle "période sémantique " de la philosophie analytique: les antiréalistes seraient plutôt à ranger parmi ses alliés. Après tout, Benoist se dit partisan d'une ligne d'analyse «frayée par Wittgens-

41. Voir sur ce point G. Sundholm, «Existence, Proof and Truth-Making: A Perspective on the Intuitionistic Conception of Truth ", Topoi, vol. I3, I994, p. I I7-I26.

42. De surcroît, s'il consacre un chapitre aux impossibilia il n'aborde pas le sujet autrement plus épineux du statut ontologique de ce qu'on pourrait appeler les possibilia, c'est-à-dire des «mondes possibles» de la sémantique de la logique modale (que ce soit les scénarios qu'on doit envisager comme solutions de remplacement pour expliquer l'occurrence d'un événement, pour comprendre les contrefactuels, etc.), statut sur lequel un débat dure depuis plusieurs décennies.

43. J. Benoist, L’adresse du réel, p. 35.

44. Ibid., p. 25.

45. Ibid., p. I06.

46. Pour ne citer que la suggestion de Yannis Moschovakis, dans « Sense and Denotation as Algorithm and Value », dans J. Oikkonen \& J. Väänänen (dir.), Logic Colloquium '90. ASL Summer Meeting in Helsinki, Berlin, Springer, I993, 2 I0-249. 
tein », qui serait celle "d'un Frege sans le platonisme ${ }^{47}$ ", or Dummett qui s'est cependant éloigné de Wittgenstein par la suite, avait frayé sensiblement le même chemin à partir des années cinquante.

La forme particulière de l'antiréalisme dummettien est certes désuète à bien des égards aujourd'hui, la "question du réalisme" s'étant déplacée autour d'un débat avec l'inférentialisme ${ }^{48}$, et mon but n'est pas non plus d'argumenter pour un retour strict à l'antiréalisme dummettien, mais simplement de suggérer de ne pas couper trop vite les ponts avec une tradition qui a aussi été très fertile en France même, dans le prolongement de la ludique de J.-Y. Girard ${ }^{49}$. Parmi les dimensions nouvelles qu'apportent ces prolongements dans le rejet de ce qu'on pourrait appeler le «tarskisme» en logique, je m'en voudrais de ne pas relever celle, très riche de sens, de l'interaction, entre autres dans l'étude du langage du point de vue de la conversation. Le langage n'a pas pour fonction première de référer mais de communiquer, en d'autres termes d'interagir dans le dialogue. Un «esprit réaliste» doit bien le savoir et ses alliés antiréalistes peuvent très amicalement lui lancer un défi: il faudrait peut-être que la phénoménologie, si on tient à ne pas l'abandonner, puisse s'accommoder de ce point de vue, où le rôle de la «deuxième personne» semble inéliminable.

47. J. Benoist, L'adresse du réel, p. I07-Io8.

48. Je fais bien sûr allusion à R. Brandom, Rendre explicite, 2 volumes, Paris, Éditions $\mathrm{du}$ Cerf, $20 \mathrm{Io}$ et L'articulation des raisons. Une introduction à l'inférentialisme, Paris, Éditions du Cerf, 2009. Les fréquentes références de Brandom à Dummett montrent bien la filiation des deux projets.

49. Pour ne prendre que deux exemples, voir l'introduction par Jean-Baptiste Joinet à J.-B. Joinet \& S. Tronçon (dir.), Ouvrir la logique au monde. Philosophie et mathématique de l'interaction, Paris, Hermann, p. 9-63; ou encore A. Lecomte, "Ludics, Dialogues and Inferentialism ", Baltic Yearbook of Cognition, Logic and Communication, vol. 8, 20I3, disponible à l'adresse: http://newprairiepress.org/biyclc/vol8/iss I/6/ 